This document is confidential and is proprietary to the American Chemical Society and its authors. Do not copy or disclose without written permission. If you have received this item in error, notify the sender and delete all copies.

\title{
A Universal Labeling Strategy for Nucleic Acids in Expansion Microscopy
}

\begin{tabular}{|r|l|}
\hline Journal: & Journal of the American Chemical Society \\
\hline Manuscript ID & ja-2021-05931b.R1 \\
\hline Manuscript Type: & Article \\
\hline Date Submitted by the & $28-J u l-2021$ \\
\hline Author: & $\begin{array}{r}\text { Wen, Gang; KU Leuven } \\
\text { Vanheusden, Marisa; KU Leuven } \\
\text { Leen, Volker; Chrometra company } \\
\text { Rohand, Taoufik; Cadi Ayyad University Faculty Polydisciplinary of SAFI, } \\
\text { Chemistry } \\
\text { Vandereyken, Katy; KU Leuven } \\
\text { Voet, Thierry; KU Leuven } \\
\text { Hofkens, Johan; Katholieke Universiteit Leuven Faculteit Wetenschappen, } \\
\text { Chemistry; Katholieke Universiteit Leuven Faculteit Psychologie en } \\
\text { Pedagogische Wetenschappen, }\end{array}$ \\
\hline
\end{tabular}

\section{SCHOLARONE ${ }^{\text {Wh }}$ Manuscripts}




\section{Introduction}

Mapping and profiling RNA in situ play a critical role in studying cellular mechanisms of gene regulation and expression, defining cell types and their functional cell states, as well as in elucidating cell-cell interactions and cellular cooperation to exert organ function. ${ }^{1-3}$ For high resolution spatial analysis of gene expression information, fluorescence in situ hybridization (FISH or ISH for dark labels) was introduced as a powerful tool for the visualization and quantification of RNA transcripts within cells and tissues. ${ }^{4,5}$ However, even though single-molecule FISH (smFISH) approaches such as multiplexed error-robust FISH (MERFISH) have significantly increased the ability to localize and quantify a large number of different transcripts, it is still difficult to discriminate the overlapping signals emitted from RNA-molecules in close proximity. ${ }^{6,7}$ As a consequence, the density of RNA-molecules that can be profiled is limited, in this way requiring to exclude highly-abundant transcripts from the detection library.
Expansion microscopy (ExM) is a recently developed technique that provides a straightforward method to achieve super-resolution imaging on conventional fluorescent microscopes. ${ }^{8-13}$ In this method, a biological signature is first covalently imprinted into a hydrogel. Next, addition of water causes swelling of the hydrogel, increasing the physical distance between grafted signature molecules in an isotropic fashion, ${ }^{14}$ as such maintaining the relative sample geometry. This allows samples to be imaged with higher resolution on diffraction limited microscopes, and furthermore reducing background signal by the intrinsic optical clearing step. Indeed, the combination of ExM with FISH (ExFISH) allows imaging ribonucleic acids with higher accuracy and higher detection efficiency as individual RNA transcripts are de-crowded after expansion of the specimen. For example, Boyden and co-workers successfully achieved super-resolution imaging of different RNA transcripts with conventional diffraction-limited microscopes via covalently attaching the sample's RNA molecules to the polymer matrix with a nitrogen mustard linker (LabelX), which allows for fluorescent detection with corresponding FISH probes post-ExM. ${ }^{14}$ Furthermore, Zhuang 
et al identified and quantified high-density RNA libraries with almost $100 \%$ detection efficiency post-expansion, comparing to less than $20 \%$ detection efficiency in unexpanded samples due to spatial overlap. ${ }^{7}$

To date, the strategies used for anchoring ribonucleic acids in ExM either directly link RNAs to the hydrogel using a chemical agent (Boyden et al. $)^{15}$ or use acrydite-modified FISH probes such as acrydite-labeled poly(dT) anchoring probes or encoding sequences, thereby grafting the target RNAs indirectly in the hydrogel (Zhuang et al, Fig. 1a). ${ }^{7}{ }^{16}$ However, as RNA molecules rather than the FISH probes are grafted to the polymer matrix, swelling the sample in water is not possible as it would induce dissociation of the FISH probes from their target. As a consequence, solutions with a certain osmolality must be used and the maximum expansion factor that can be achieved is limited. Moreover, acrydite-labeled poly(dT) oligonucleotides can only be used to link intact polyadenylated RNA molecules to the gel matrix. In addition to these strategies, an approach where both grafting and reporting functionality are combined in the same oligonucleotide probe was also reported (Mueller et al.). ${ }^{17}$ However, even though the price of every functionalized and short $(n<100)$ synthetic oligonucleotide is acceptable, such RNA FISH experiments often require dozens or hundreds of different sequences to cover transcripts and attain signal amplification. ${ }^{17,18}$ Therefore, although the introduction of fluorescent dyes and reactive groups is offered by commercial partners, cost becomes prohibitive for multiplexed analysis.

To resolve these limitations, we investigate chemical methods for labeling oligonucleotides, for use in optical clearing microscopy and expansion microscopy, and this extended to multivalent reagents (Fig. 1b). In this report, we design and evaluate a series of chemical probes that allow post-synthetic modification of DNA oligonucleotide libraries for RNA detection, introducing labeling and anchoring functionalities to the ribonucleic acids in a single step. Elaborating on our recently described TRITON approach, ${ }^{19}$ where ExM has been used to achieve nanoscale imaging of cytoskeleton and lipid membranes via simultaneous targeting, labeling and grafting of biomolecules in one step, we synthesized a series of novel TRITON linkers for targeting ribonucleic acids in ExM. We first validate their application in oligonucleotide-conjugated antibodies. After validation, we then evaluate the performance of such molecules for labeling and anchoring RNA molecules in ExM.

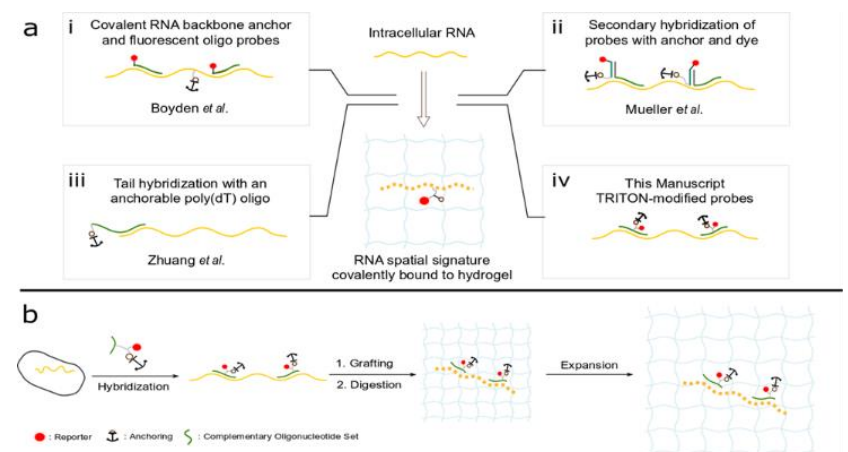

Figure 1. Schematic diagram of the different approaches for grafting and imaging ribonucleic acids within the ExM polymer. (a) The anchoring strategies of RNA via LabelX (i), acrydite-modified FISH probes (ii), acrydite-modified poly(dT) oligonucleotides for tail hybridization (iii) and our TRITON-modified encoding sequences (iv). (b) Workflow of our TRITON linkers used in ExM.
Encoding sequences are labeled with our TRITON molecules in a single step approach.

\section{Results and Discussion}

Construction of the chemical labeling agents. Building on an extensive body of literature on the use of ribonucleic acid probes in biotechnology, ${ }^{20-28}$ we synthesized a library of oligonucleotide probes, combining a fluorescent reporter with a reactive group for oligonucleotide covalent binding in the ExM matrix. We selected different chemical functionalities, including photo-activated and thermal-activated reactive groups (Structures in Fig. 2a), and we then evaluated and optimized labeling efficiency of these direct labeling agents against oligonucleotides in different mild conditions using spectrophotometry, including exploring different equivalents of labeling agents, reaction time, and reaction buffers as well as heating or UV irradiation (Details in Table S1). Interestingly, for thermal-activated groups, the nitrogen mustard containing compound $\mathbf{1} \mathbf{j}$, as used previously in ExM, resulted in lower labeling density in a range of conditions compared to the platinum(II)-based compound $1 \mathbf{i}$ in the same conditions (Labeling density: dye numbers per oligonucleotide, entry 26-27 vs entry 30-32 in Table S1). For instance, compound 1i labeled oligonucleotides with a labeling density of 2.57 , comparing to 2.14 of nitrogen mustardmodified oligonucleotides (Entry 27 and 32 in Table S1). For photo-activated functionalities, aryl azide (phenyl azide and meta-nitrophenyl azide) labeled oligonucleotides with high efficiency, reaching a labeling density of $\sim 4$ per oligonucleotide (Entry 1-16 in Table S1). In addition, we observed how a positively charged "spacer arm" significantly improved the agents' ability to react with the negative charged oligonucleotides via comparing labeling efficiency of compound $\mathbf{1 b}$ with $\mathbf{1 a}$ under the same conditions (e.g. labeling density: 3.84 vs 1.20 , entry 4 and 1 in Table S1).

With this set of reagents in hand, we selected prime candidates for assessment of the influence on hybridization properties and labeling approaches, optimizing their use in biological applications (Fig. 2b-2c and Fig. S1). To evaluate whether reagent-labeled oligonucleotides could still hybridize with their complementary oligonucleotides, we first performed an immunofluorescence staining of microtubules, where we used secondary antibodies conjugated with a docking strand for a complementary short fluorescent oligonucleotide. Next, the complementary fluorescent oligonucleotides (i.e. the readout probes) were functionalized with the reagents described, and followed by intracellular hybridization to the antibody target, with the signal detected using a confocal microscope. Among photo-reactive groups, we chose meta-nitrophenyl azide as a functional group, as this can be activated at longer wavelengths, thus avoiding UV light induced damage to the oligonucleotide. ${ }^{29,30}$ As a thermal-reactive group, the $\mathrm{Pt}(\mathrm{II})$ linker was selected in view of the higher degree of labeling density achieved over nitrogen mustards. From our comparative analysis, we found that the specimen stained by meta-nitrophenyl azide-modified readout probes had to be imaged with higher laser power than the platinum(II)-contained specimen $(31 \mu \mathrm{W}$ and $22 \mu \mathrm{W}$ vs $4 \mu \mathrm{W}$ and $2 \mu \mathrm{W}$, Fig. 2d-2g respectively), indicating a reduced hybridization efficiency when readout probes were labeled by meta-nitrophenyl azide. In contrast, platinum(II)-modified oligonucleotides showed excellent hybridization efficiency at a labeling density of 1-2 labels per oligonucleotide; and hybridization ef- 
ficiency would be slightly lower at a labeling density of 3-4 labels per oligonucleotide via comparing mean fluorescence intensity with a control experiment where a single dye was introduced at the 5 '-end of the oligonucleotide (Fig. S1).

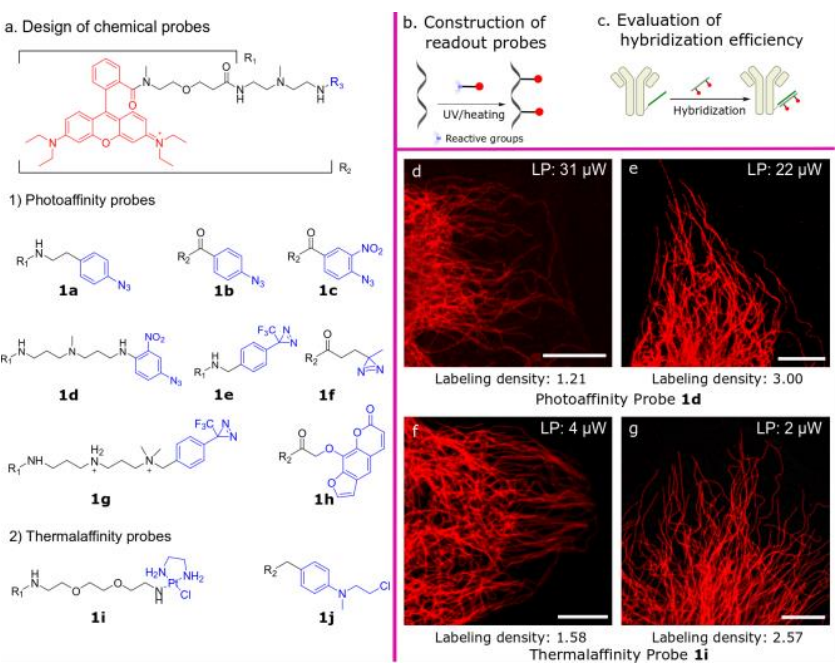

Figure 2. Design and optimization of chemical probes for nucleic acids. (a) Synthesis of a series of probes with different functional groups. (b) Schematic representation of the construction of readout probes. (c) Schematic diagram of evaluating hybridization properties via combination with oligonucleotide-labeled antibodies. (d-g) Images obtained in immunostaining experiments with compound 1d and 1i. All experiments are performed in the same condition except the laser power used for imaging. Scale bars: $10 \mu \mathrm{m}(\mathrm{d}, \mathrm{e}, \mathrm{f}$, g).

From this optimized scaffold, additional functionality was introduced to the reagents to allow grafting to the polymeric matrix and use in expansion microscopy. Hence, we designed and synthesized a series of trifunctional molecules for nucleic acids, including: a reporter part, a polymerizable moiety, and a targeting unit (Structures in Fig. 3). Fluorescent dyes were selected in line with our previous analysis of dye survival during the polymerization step, (Rhodamine B, Rhodamine 6G, both with good synthetic accessibility and good survival rate during the polymerization step). ${ }^{19}$ Non-fluorescent labels included reactive groups such as azide and alkyne, and this for flexible labeling approaches with post-polymerization staining. ${ }^{31}$ As platinum(II)-based reagents attack N7 position of guanine moieties, resulting in no discriminatory effect between different nucleic acid backbones, we considered these reagents as a universal labeling method for nucleic acids. ${ }^{23,25,32}$ Finally, this effort resulted in a set of multifunctional reagents that were forwarded for further use.

Direct grafting of oligonucleotide reporter probes. We first assessed the performance of our reagents in the abovementioned regular immunostaining protocol, now also including polymerization and sample expansion. Our TRITON compound 2a-conjugated readout probes could be efficiently hybridized to docking strand-labeled secondary antibodies (Fig. S2) and thus resulted in a clear fluorescent signal for microtubules in different ExM modalities (Fig. 3). Comparing to the obtained resolution of $287 \pm 28 \mathrm{~nm}$ (mean \pm standard deviation, $\mathrm{n}=49$ ) in preexpansion, we achieved a resolution of $105 \pm 9 \mathrm{~nm}$ (mean \pm standard deviation, $n=49$ ) after $\times 4$ expansion, calculated using the full width at half-maximum (FWHM) when considering the obtained expansion factor of 3.66 (Fig. 3c-3f, Fig. S3-5). This experimental resolution is consistent with the theoretical resolution of $94 \mathrm{~nm}$ that can be achieved for this type of experiment based on the Rayleigh criterion. In $\times 10 \mathrm{ExM}$, an expansion factor of 7.60 enabled us to achieve an improved resolution of 50 $\pm 6 \mathrm{~nm}$ (mean \pm standard deviation, $\mathrm{n}=49$ ), again in line with the theoretical resolution of $46 \mathrm{~nm}$ that can be achieved for the used experimental settings (Fig. 3g-3j, Fig. S3 and S7 vs $283 \pm$ $28 \mathrm{~nm}$ (mean \pm standard deviation, $\mathrm{n}=49$ ) obtained in pre-expansion, Fig. S6). Similar results were obtained for "dark" labels, onto which we introduced dyes after digestion through a DBCO-azide click reaction (Fig. 3k-3o). This method effectively avoids the destruction of dyes during the polymerization step, extending the dye scope that can be used in ExM, such as the use of Cy5 (Fig. 3n-3o). Furthermore, it should be noted that such reagents are also adequate stains for direct functionalization of protein targets, extending on our previous labeling concepts (Fig. S8). ${ }^{19}$
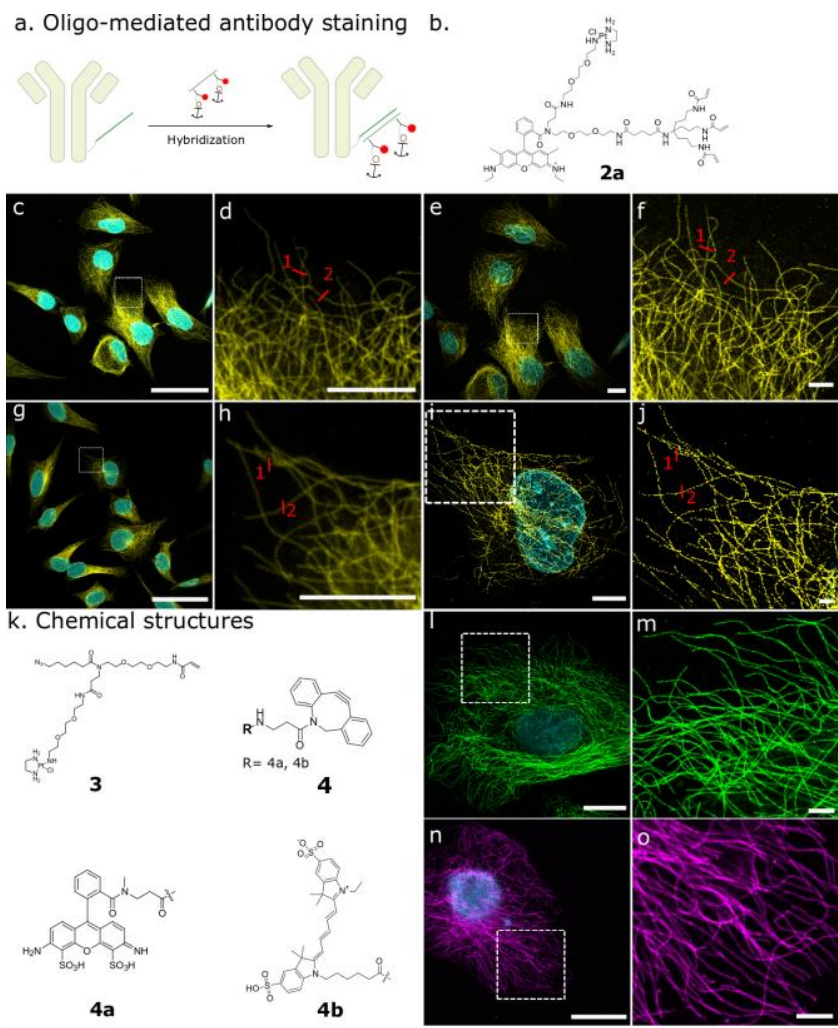

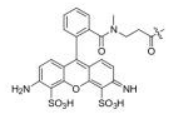

$4 a$

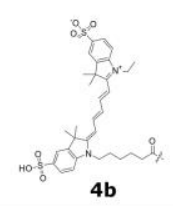

Figure 3: The immunostaining experiment using TRITON-based readout probes in $\times 4$ and $\times 10$ ExM. (a) Oligonucleotide-mediated immunostaining in which imager strands were labeled by our TRITON linker. (b) Chemical structure of TRITON linker 2a. (c-f) Immunostaining against microtubules with TRITON linker $\mathbf{2 a}(\mathrm{Rh}$ $6 \mathrm{G}$, platinum and acrylates) in $\times 4$ ExM. (c) Pre-expansion image obtained in $\times 4$ ExM. (d) Zoom of the highlighted region in (c) and representative FWHM line profiles (1) $355 \mathrm{~nm}$ and (2) $229 \mathrm{~nm}$. (e) Post-expansion image obtained in $\times 4$ ExM. (f) Zoom of the highlighted region in (e) and representative FWHM line profiles (1) 419 $\mathrm{nm}$ and (2) $345 \mathrm{~nm}$. (g-j) Immunostaining against microtubules with TRITON linker 2a in $\times 10$ ExM. (g) Pre-expansion image obtained in $\times 10$ ExM. (h) Zoom in on highlighted region in (g) and representative FWHM line profiles (1) $269 \mathrm{~nm}$ and (2) $292 \mathrm{~nm}$. (i) Postexpansion image obtained in $\times 10$ ExM. (j) Zoom in on highlighted region in (i) and representative FWHM line profiles (1) $347 \mathrm{~nm}$ and (2) $407 \mathrm{~nm}$. (k) Chemical structures of compound $\mathbf{3}$ and $\mathbf{4}$ used in 
a post-digestion labeling strategy. (1) Post-digestion labeling image obtained with compound 3 and compound $\mathbf{4 a}$ (Atto 488-DBCO) in $\times 4$ ExM. (m) Zoom in on highlighted region in (1). (n) Post-digestion labeling image obtained with compound 3 and compound $\mathbf{4 b}$ (Sulfo-Cy5-DBCO) in $\times 4$ ExM. (o) Zoom in on highlighted region in (n). Scale bars: $50 \mu \mathrm{m}(\mathrm{c}, \mathrm{e}, \mathrm{g}, \mathrm{i}, \mathrm{l}, \mathrm{n}), 10 \mu \mathrm{m}(\mathrm{d}, \mathrm{f}, \mathrm{h}, \mathrm{j}, \mathrm{m}, \mathrm{o})$.

Direct oligonucleotide labeling for RNA FISH in ExM. We set out to test the performance of our newly developed TRITON linkers for detecting RNA transcripts post-expansion. To evaluate retention of the RNA probes post-ExM, we conjugated hybridization chain reaction (HCR) v3.0 split initiator probes against phosphoglycerate kinase 1 ( $P G K 1$ split initiator probe sequences and amplification sequences can be found in the original manuscripts ref 33 and 34 respectively) ${ }^{33,34}$ to a TRITON linker with rhodamine 6G (Compound 2a). Average labeling density of TRITON linker per probe sequence of $P G K 1$ transcripts was 2.08 and the concentration of labeled sequences was $17 \mu \mathrm{M}$. Upon hybridization to their cellular target and matrix grafting, single mRNA molecules were clearly visible preExM (Fig. S9 a-b and e-h). However, signals fell below the detection limit post-ExM (Fig. S9 c-d). This is due to the volumetric signal dilution and dye degradation (Rhodamine 6G: survival rate approximately $40 \%),{ }^{19}$ both of which are intrinsic to the expansion procedure. Such low signal to noise for RNAFISH is commonly observed, also outside the context of ExM, yielding false-positive and false-negative detections. ${ }^{5,}{ }^{17}$ Hence, to account for the low signals in ExM, we extended the use of our optimized polynucleotide grafting reagents to signal amplification methods. We amplified the fluorescent signal postExM by making use of initiator sequences, which trigger HCR while enabling automatic background suppression (Fig. 4a). ${ }^{33}$, ${ }^{35} \mathrm{We}$ first evaluated to what extent TRITON-labeled encoding probes could still specifically bind to their mRNA targets. To address this, we compared the number of $P G K 1$ transcripts detected in our TRITON samples in which the encoding sequences were modified with TRITON linker $\mathbf{2 a}$ with the number of PGK1 transcripts in a control experiment where the encoding sequences were without modification of our TRITON linker 2a. We found that the mean number of detected PGK1-mRNA was very similar between these two groups ( 262 per cell in the control experiment vs 257 per cell in our TRITON samples, Fig. 4b and Table S2). Overall, these results indicate that attachment of our TRITON linkers to the RNA detection probes does not hinder the probe's binding ability and specificity.

To assess the anchoring yield of the TRITON linker-coupled FISH probes in ExM, the ratio of the detected transcripts for a same cell post- over pre-expansion was calculated. Several post-ExM 2D images were compared to a single pre-ExM 2D image to compensate for the expansion in the 3rd dimension and the amount of detected transcripts was evaluated (TrackMate Fiji plugin). We found no post-expansion loss of transcript spots which were detected pre-expansion when comparing cells $(\mathrm{n}=$ 10 ) in three independent samples (Fig. 4c-4g and Fig. S10). Recovery percentages above $100 \%$ can be attributed to the decrowding of molecules in dense areas, where the spots will overlap in the pre-expansion image, resulting in an underestimation of the amount of spots. Additionally, an improved signal-to-noise ratio is clearly observed after expansion, owing to the intrinsic optical clearing and thus a reduced autofluorescence signal. ${ }^{17,36}$
Considering that some dyes, e.g. cyanines, would be completely destroyed during polymerization, an alternative routine was to introduce these dyes after the sample digestion step. GAPDH was chosen as an example to evaluate our post-labeling strategy, in which an Alexa 647-labeled hairpin was applied only after digestion (Fig. 4h-4i, GAPDH probe and amplification sequence information $\left.{ }^{33}, 34\right)$. It should be noted that average labeling density of TRITON linker per probe sequence of GAPDH transcripts was 1.72 and the concentration of labeled sequences was $17 \mu \mathrm{M}$. Additionally, these compounds allow for rapid functionalization of DNA oligonucleotide libraries for multicolor analysis in ExM, as evidenced by the simultaneous detection of $P G K 1$ and $G A P D H$ transcripts, by using two different encoding sequences, each grafted to the polymer matrix with the TRITON linker $\mathbf{2 a}$ (Fig. $\mathbf{4 j - 4 m}$ ). In this two-color staining experiment, considering the variation in transcript abundance, ${ }^{15}$,

${ }^{37}$ PGK1 and GAPDH transcripts are selected to prove that our approach is also able to capture a broad concentration range of transcripts.
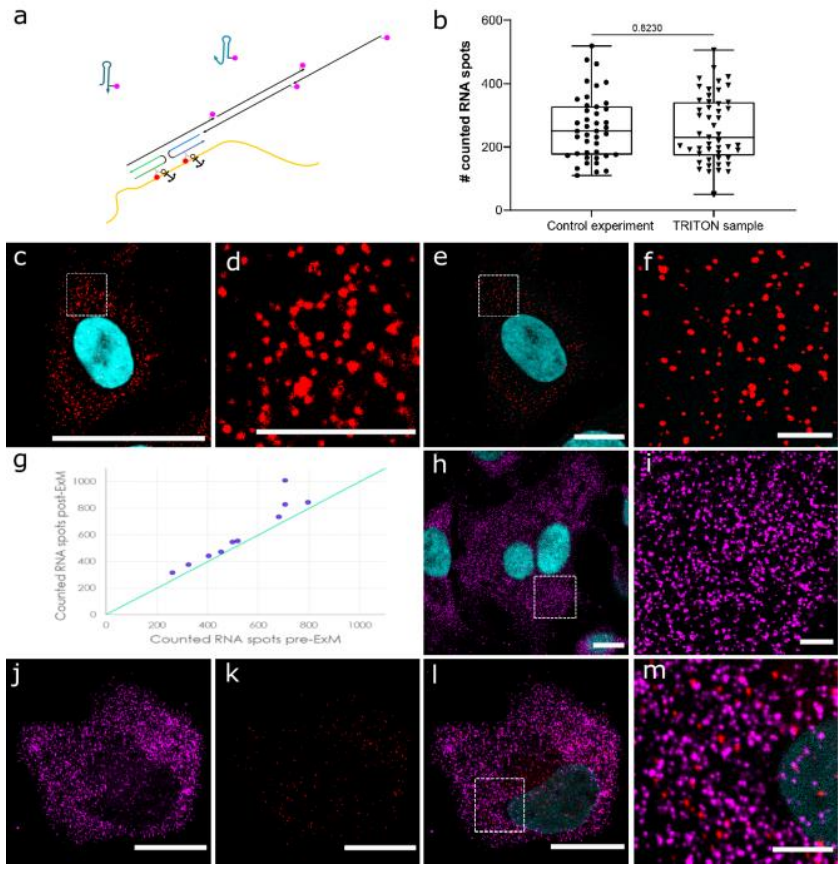

Figure 4. mRNA detection via combining ExM with HCR v3.0 using TRITON linker 2a. (a) Design of HCR v3.0-mediated signal amplification. TRITON-modified FISH probes first hybridize to the target mRNA and metastable DNA hairpins bearing fluorophores then bind to the split initiators, thus triggering signal amplification. (b) Estimated number of $P G K 1$ transcripts per cell in a control experiment and our TRITON samples in three independent experiments. The $\mathrm{P}$ value is listed, and $\mathrm{P}<0.05$ is considered significant. Control experiment: Commercial encoding probes (no TRITON linker 2a modification) directly bind to PGK1-mRNA and Alexa Fluor 594-labeled hairpins then bind to the split initiators to trigger signal amplification. (c-f) Images of $P G K 1$ before and after expansion, using Alexa Fluor 594-labeled hairpins. (c) Preexpansion image of PGK1 transcripts. (d) Zoom in on highlighted region in (c). (e) Post-expansion image of the same cell. (f) Zoom in on highlighted region in (e). (g) mRNA molecules counted before versus after expansion for cells from 3 independent samples $(\mathrm{n}=10)$. The cyan line is used as a guide to prove the loss of $P G K 1$ transcripts detected in unexpanded samples. (h) Post-labeling image of GAPDH transcripts, using Alexa Fluor 647-labeled hairpins. (i) Zoom in on highlighted region in (h). (j-m) Multi-color 
staining against $P G K 1$ and $G A P D H$ transcripts in Hela cells (PGK1: Alexa Fluor 594-labeled hairpins; GAPDH: Alexa Fluor 647-labeled hairpins); nuclei were marked with DAPI. (j) Image of $G A P D H$. (k) Image of $P G K 1$. (1) Merge image of $G A P D H$ and $P G K 1$. (m) Zoom in on highlighted region in (l). Scale bars: 50 $\mu \mathrm{m}(\mathrm{c}, \mathrm{e}, \mathrm{h}, \mathrm{j}, \mathrm{k}, \mathrm{l}), 10 \mu \mathrm{m}(\mathrm{d}, \mathrm{f}, \mathrm{i}, \mathrm{m})$.

\section{Conclusion}

In this work, we design and synthesize a series of novel di/multivalent molecules (e.g. TRITON 2a, 3 and compound S27) that allow for rapid functionalization of DNA oligonucleotides as probes for expansion microscopy, in a single step approach. From the design, the degree of labeling per oligonucleotide and functionality imparted to the oligonucleotide can easily be tuned to match the labeling requirements. As such, the TRITON-functionalized oligonucleotides are compatible with different labeling strategies in the immunofluorescence staining of microtubules, such as a direct conjugation of biomolecules with dyes or a post-digestion labeling strategy via a click reaction, effectively avoiding signal loss during polymerization and digestion steps. We demonstrate the use of such multivalent reagents to detect and highlight the location and origin of oligonucleotide conjugated antibodies as well as mRNA, for use in optical clearing microscopy and ExM. We validate that mRNA can be detected with high specificity and sensitivity via the combination of our reagents with existing RNA FISH amplification and multiplexing approaches. Through combination of these reagents with our previously described methods for direct grafting of biomolecule locations into a polymeric matrix, we expect their adoption in the simultaneous analysis of a range of biological targets within their cellular or tissue context.

\section{Methods}

Optimization of labeling procedures for oligonucleotides. To a solution of $1.5 \mu \mathrm{L}$ ssDNA (2.67 mM in 1x PBS, readout strand: AAA AGA TCC GAT TGG AAC CGT CCC AAG

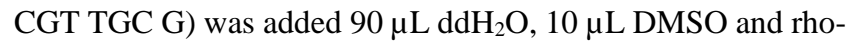
damine $\mathrm{B}$ with different functional groups (Structures in Fig. 2a). Reaction conditions were varied in line with Table S1, and resulting labeled oligonucleotides were purified by Nap-5 column (GE Healthcare, USA) using the manufacturer's protocol

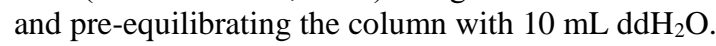

Evaluation of labeling density of oligonucleotides. As dyes contribute to the absorbance at $260 \mathrm{~nm}$, concentration of oligonucleotides should be corrected with a certain correction factor (C.F.260), which is a constant value determined by dividing the absorbance of the free dye at $260 \mathrm{~nm}$ with the absorbance of the free dye at the peak wavelength $\left(\lambda_{\max }\right)$ : C.F.260 $=A_{260}$, free dye $/ \mathrm{A}_{\lambda \max }$, free dye. The labeling ratio is then calculated as follow: $n_{\text {dye }} / n_{\text {oligo }}=\left(A_{\text {dye }} \times \varepsilon_{\text {oligo }}\right) /\left(A_{\text {oligo }} \times \varepsilon_{\text {dye }}\right)$ and $A_{\text {oligo }}=A_{260}-\left(A_{\text {dye }}\right.$ $\times C . F .260)$.

Cell culture. HeLa cells (ATCC) were cultured at $37^{\circ} \mathrm{C}$ in a 5 $\% \mathrm{CO}_{2}$ humidified atmosphere in high glucose $(4.5 \mathrm{~g} / \mathrm{L})$, glutamine free, phenol red-free Dulbecco's Modified Eagle Medium (DMEM; Life Technologies) supplemented with $10 \%$ (v/v) fetal bovine serum, $50 \mu \mathrm{g} / \mathrm{mL}$ gentamicin (Life technologies) and $1 \%$ glutamax. When cells reached $70 \%$ confluency, they were washed with 1x DPBS (no calcium, no magnesium; Life Technologies) before detaching with 10x TrypLETM Enzyme (Life Technologies). Afterwards, cells were seeded into the correct imaging chambers depending on the experiment.

Sample fixation and immunofluorescence. HeLa cells were seeded onto size $22 \mathrm{~mm}$ x $22 \mathrm{~mm} \# 1.5$ coverglasses at a density of $40 \times 10^{3}$ cells $/ \mathrm{cm}^{2}$ and cultured as mentioned in the previous section. The next day, cells were extracted twice for 30 seconds with pre-heated MTSB (PEM Triton X, 80 mM PIPES pH 6.8, $5 \mathrm{mM}$ EGTA, $1 \mathrm{mM} \mathrm{MgCl}_{2}, 0.5 \%$ Triton X-100). Then, the sample was fixed in MTSB solution containing $0.5 \%$ glutaraldehyde for 10 minutes and washed three times for 5 minutes with PBS before quenching with $0.2 \%$ sodium borohydride in PBS at room temperature for 7 minutes. Cells were washed three times with PBS and then blocked $2 \mathrm{x}$ for 5 minutes with blocking buffer (PBS, 1\% BSA). Next, coverslips were incubated with primary antibodies (mouse anti $\alpha$-tubulin, Abcam, ab7291) in staining buffer (PBS, 3\% BSA) at a concentration of $2 \mu \mathrm{g} / \mathrm{mL}$ for 1 hour at room temperature and washed with PBS three times for 5 minutes each. Specimens were incubated with DNA-conjugated antibodies (Goat Anti-Mouse IgG, Abcam ab) for 1 hour in staining buffer with a dilution of 1:50. After incubation, samples were washed $2 \mathrm{x}$ with blocking buffer for 5 minutes and further washed once with PBS. After that, samples were incubated in hybridization wash buffer ( $20 \%$ formamide in 2x SSC) at room temperature for $10 \mathrm{~min}$ and incubated overnight with $10 \mathrm{nM}$ readout oligonucleotides labeled with TRITON linker in hybridization buffer (20\% formamide, $10 \%$ dextran sulfate, $0.1 \%$ Tween-20 in 2x SSC) supplemented with $0.2 \mathrm{mg} / \mathrm{mL}$ sheared salmon sperm DNA in an airtight container at $37^{\circ} \mathrm{C}$. After hybridization, samples were washed three times for 30 min with hybridization wash buffer at $37{ }^{\circ} \mathrm{C}$. Finally, specimens were stained with $1 \mu \mathrm{g} / \mathrm{mL}$ DAPI and then washed three times with PBS.

Sample fixation and RNA FISH staining. HeLa cells were seeded onto size $22 \mathrm{~mm}$ x $22 \mathrm{~mm} \# 1.5$ coverglasses at a density of $40 \times 10^{3}$ cells $/ \mathrm{cm}^{2}$ and cultured as mentioned in the previous section for $24 \mathrm{~h}$. Culture medium was removed and washed once with $1 \mathrm{~mL}$ PBS. It was fixed with $1 \mathrm{~mL} \mathrm{4 \%} \mathrm{formaldehyde}$ at room temperature for $10 \mathrm{~min}$. Next, the sample was washed twice with $1 \mathrm{~mL}$ PBS and then permeabilized with $1 \mathrm{~mL} 70 \%$ ethanol at $-20{ }^{\circ} \mathrm{C}$ overnight. After that, ethanol was removed and the sample was air-dried at room temperature and washed twice with $2 \mathrm{~mL} \mathrm{2x} \mathrm{SSC}$. Afterwards, the sample was first incubated with $100 \mu \mathrm{L}$ probe hybridization buffer(PHB, $30 \%$ formamide, 5x SSC, $9 \mathrm{mM}$ citric acid pH 6.0, 0.1\% Tween 20, $50 \mu \mathrm{g} / \mathrm{mL}$ heparin, $1 \mathrm{x}$ Denhardt's solution and $10 \%$ dextran sulfate) at $37{ }^{\circ} \mathrm{C}$ for $30 \mathrm{~min}$ in a humidified chamber and then incubated overnight with another $100 \mu \mathrm{L}$ PHB supplemented with $10 \mathrm{nM}$ of each encoding probe in a humidified chamber. The specimen was washed four times with $300 \mu \mathrm{L}$ probe wash buffer (30\% formamide, 5x SSC, $9 \mathrm{mM}$ citric acid $\mathrm{pH}$ 6.0, 0.1\% Tween 20 and $50 \mu \mathrm{g} / \mathrm{mL}$ heparin), 5 min each time. It was further washed twice for 5 min with $300 \mu \mathrm{L} 5 \mathrm{x}$ SSCT ( $5 \mathrm{x}$ sodium chloride sodium citrate and $0.1 \%$ Tween 20 ), and incubated with $100 \mu \mathrm{L}$ probe amplification buffer (5x SSC, $0.1 \%$ Tween 20 and $10 \%$ dextran sulfate) at room temperature for $30 \mathrm{~min}$. Meanwhile, $3 \mu \mathrm{L}$ of each hairpin stock $(3 \mu \mathrm{M})$ was snapcooled by heating to $95{ }^{\circ} \mathrm{C}$ for 90 seconds using a PCR thermocycler, and cooling them down to room temperature for $30 \mathrm{~min}$. After 
that, the sample was incubated for 45 minutes at room temperature with $100 \mu \mathrm{L}$ probe amplification buffer supplemented with $4.5 \mathrm{nM}$ of each hairpin stock ( $P G K 1$ : Alexa Fluor 594labeled B1h1 and B1h2). After incubation, the sample was washed once for $5 \mathrm{~min}$ with $300 \mu \mathrm{L} 5 \mathrm{x}$ SSCT, and was then incubated with $600 \mu \mathrm{L} 5 \mathrm{x}$ SSCT supplemented with $1 \mu \mathrm{g} / \mathrm{mL}$ DAPI for $10 \mathrm{~min}$. Finally, the specimen was washed three times for 5 min with $300 \mu \mathrm{L} \mathrm{5x} \mathrm{SSCT.}$

Gelation, Digestion and Expansion. For $\times 4$ ExM, gelation solution was prepared with $2 \mathrm{M} \mathrm{NaCl}, 8.625 \%$ (w/w) sodium acrylate, $2.5 \%(\mathrm{w} / \mathrm{w})$ acrylamide, $0.15 \%(\mathrm{w} / \mathrm{w})$ N,N'-methylenebisacrylamide and $0.01 \%$ 4-hydroxy-TEMPO in PBS supplemented with $0.15 \%$ tetramethylenediamine (TEMED) and $0.15 \%$ ammonium persulfate (APS) and kept on ice until further use. For $\times 10$ ExM, monomer solution was prepared with 1.335 g N,N-dimethylacrylamide (DMAA), $0.32 \mathrm{~g}$ sodium acrylate (SA) and $2.85 \mathrm{~g}$ deionized water. The solution was bubbled for $40 \mathrm{~min}$ at room temperature with nitrogen gas to remove the molecular oxygen. Potassium persulfate (KPS) solution $(0.1 \mathrm{ml}$ freshly prepared, $36 \mathrm{mg} / \mathrm{mL}$ ) was added to $0.9 \mathrm{~mL}$ monomer solution and the mixture was further purged with nitrogen gas for $15 \mathrm{~min}$ on ice. After that, $4 \mu \mathrm{L}$ TEMED was added to the mixture to accelerate the polymerization process. Samples were first washed with $80 \mu \mathrm{L}$ gelation solution and gelation was performed with another $80 \mu \mathrm{L}$ fresh gelation solution on a sigmacote ${ }^{\circledR}$-treated glass slides on which two size $22 \mathrm{~mm} \times 22 \mathrm{~mm}$ no. 1.5 coverslips were placed (spaced by $\pm 1 \mathrm{~cm}$ ) to define the edges of the gelation chamber. Samples were then transferred to a humidified chamber and incubated at $37^{\circ} \mathrm{C}$ for $1.5 \mathrm{~h}$. After gelation, the sample was carefully detached from the glass slide and was trimmed to the desired shape. Next, the hydrogel was digested by incubation in $1.5 \mathrm{~mL}$ of proteinase $\mathrm{K}$ (New England Biolabs) diluted to $8 \mathrm{U} / \mathrm{mL}$ in digestion buffer $(50 \mathrm{mM}$ Tris $(\mathrm{pH}$ 8.0), 1 mM EDTA, $0.5 \%$ TritonX-100, $0.8 \mathrm{M}$ guanidine $\mathrm{HCl}$ ) overnight at RT for $\times 4$ ExM and overnight at $37{ }^{\circ} \mathrm{C}$ for $\times 10$ ExM. And then gels were stained with $1 \mu \mathrm{g} / \mathrm{mL}$ DAPI and washed three times with PBS. Finally, microtubule-stained gels were expanded in $\mathrm{ddH}_{2} \mathrm{O}$; for RNA FISH, gels were expanded with $0.05 x$ SSC.

Post-expansion detection of RNA FISH. Gelated specimens for RNA FISH were prepared as mentioned above. After digestion, gels were washed three times for 5 min with $300 \mu \mathrm{L} 2 \mathrm{x}$ SSC and then washed twice for 5 min with $300 \mu \mathrm{L} 5 \mathrm{x}$ SSCT, 5 $\mathrm{min} /$ time. Next, gels were incubated with $100 \mu \mathrm{L}$ probe amplification buffer at room temperature for $30 \mathrm{~min}$. Meanwhile, 3 $\mu \mathrm{L}$ of each hairpin stock $(3 \mu \mathrm{M})$ was snapcooled and next, gels were incubated with $200 \mu \mathrm{L}$ probe amplification buffer supplemented with the snapcooled hairpin stock at room temperature for 45 min (PGK1: Alexa Fluor 594-labeled B1h1 and B1h2; GAPDH: Alexa Fluor 647-labeled B3h1 and B3h2). Gels were then washed once for 5 min with $300 \mu \mathrm{L} 5 \mathrm{x}$ SSCT and incubated with $600 \mu \mathrm{L} 5 \mathrm{x}$ SSCT supplemented with $1 \mu \mathrm{g} / \mathrm{mL}$ DAPI for $10 \mathrm{~min}$. Gels were further washed three times for $5 \mathrm{~min}$ with $300 \mu \mathrm{L} 5 \mathrm{x}$ SSCT. Finally, gels were expanded with 0.05x SSC.

Post-expansion detection of immunostained microtubules. Gelated specimens for immunofluorescence were prepared as mentioned above. After digestion, gels were rinsed with 1x PBS once and then stained with Atto 488-DBCO or
Sulfo-Cy5-DBCO (5 $\mu \mathrm{M}$ in PBS) for $1 \mathrm{~h}$ at $37{ }^{\circ} \mathrm{C}$. Next, specimens were stained with $1 \mu \mathrm{g} / \mathrm{mL}$ DAPI and then washed three times with PBS. Finally, specimens were expanded in $\mathrm{ddH}_{2} \mathrm{O}$.

\section{Sample mounting}

1. Re-embedding of expanded hydrogels. For microtubule staining, the expanded gel was re-embedded in a charge-neutral polyacrylamide gel to stabilize the gel. For this, the digested gel was transferred to a 6 well plate (Cellvis, Product \#:P06-1.5H$\mathrm{N})$ of which the glass surface was treated with bind-silane solution ( $4 \mathrm{~mL}$ of ethanol, $100 \mu \mathrm{L}$ of acetic acid, $3 \mu \mathrm{L}$ of bind-silane, $900 \mu \mathrm{L}$ of MQ) at room temperature for $1 \mathrm{~h}$ and then expanded four times with $\mathrm{ddH}_{2} \mathrm{O}, 15 \mathrm{~min}$ each time. The expanded gel was quickly rinsed with $2 \mathrm{~mL}$ re-embedding solution and further incubated in another fresh $2 \mathrm{~mL}$ re-embedding solution for 20 min (re-embedding solution: $3 \%$ acrylamide, $0.15 \% \mathrm{~N}, \mathrm{~N}^{\prime}-$ Methylenebisacrylamide and $5 \mathrm{mM}$ Tris $\mathrm{pH} 10.5$ supplemented with $0.05 \%$ APS and $0.05 \%$ TEMED). After that, the solution was removed from the gel and a \#1.5 $22 \mathrm{~mm} \times 22 \mathrm{~mm}$ was placed on the top of gel. Next, the plate was transferred to a container purged with nitrogen and then incubated at $37{ }^{\circ} \mathrm{C}$ for $1.5 \mathrm{~h}$. Finally, the gel was washed three times for $30 \mathrm{~min}$ with $\mathrm{ddH}_{2} \mathrm{O}$ and the coverslip was easily removed from the sample after the second washing step.

2. Sample mounting with poly-D-lysine. For RNA FISH experiments, the expanded gel was mounted on a poly-D-lysinecoated 6 well plate to reduce gel drift during image acquisition. Briefly, glass surface of the 6 well plate was coated with $1 \mathrm{~mL}$ $0.1 \%$ (w/v) poly-D-lysine in water for $20 \mathrm{~min}$. The solution was then removed and the coated glass surface was rinsed three times with deionized water. Next, the surface was air-dried for 1 hour. After that, the expanded gel was transferred to the coated surface and a coverslip was placed on the top of the gel. Finally, the gel was rinsed with $0.5 \mathrm{~mL} 0.05 \mathrm{x}$ SSC and the top coverslip was removed. It is worth mentioning that specimens stained with sulfo-Cy5 were mounted with poly-D-lysine as sulfo-Cy5 would be destructed during the re-embedding protocol.

Conjugating antibodies with DNA docking strands. DNA-labeled secondary antibodies were prepared based on the protocol below. In brief, to a solution of $25 \mu \mathrm{L}$ thiolated DNA ( $1 \mathrm{mM}$ in 1x PBS, docking strand: HS- CGC AAC GCT TGG GAC GGT TCC AAT CGG ATC TTT T), $55 \mu$ L EDTA buffer ( $1 \mathrm{mM}$ EDTA in $1 \mathrm{x}$ PBS, $\mathrm{pH} 8)$ and $20 \mu \mathrm{L}$ DTT (500 mM in EDTA buffer) was added and incubated at room temperature for 2 hours on a thermal shaker. Then, the sample was purified by a Nap-5 column (GE Healthcare, USA) pre-equilibrated with 10

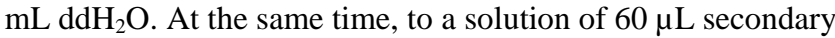
antibody (2 $\mathrm{mg} / \mathrm{mL})$, a solution of maleimide-PEG2succinimidyl ester $(2.4 \mu \mathrm{L}$, as a DMF solution $(4 \mathrm{mM}))$ was added in a 1:10 molar ratio, and was incubated for $90 \mathrm{~min}$ at 4 ${ }^{\circ} \mathrm{C}$. After incubation, antibodies were purified using a $0.5 \mathrm{ml} 40$ kDa Zeba desalting column (ThermoFisher \#87766), with PBS as eluent. Next, the activated antibody is combined with the functionalized oligonucleotides in a 1:10 molar ratio and reacted for 40 minutes at room temperature, followed by purification using Amicon Ultra $0.5 \mathrm{~mL} 50 \mathrm{kDa}$ Centrifugal Filters (EMD Millipore \#UFC510096), washing several times with 1x PBS. The functionalized antibodies are stored at $4{ }^{\circ} \mathrm{C}$ for 2 
weeks or snap-frozen aliquots were prepared and stored at -80 ${ }^{\circ} \mathrm{C}$ for long term storage.

Labeling readout strands with TRITON linker. TRITONmodified readout probes were prepared based on the aforementioned protocol. In brief, to a solution of $1.5 \mu \mathrm{L}$ non-functionalized DNA (2.67 mM in 1x PBS, readout strand: AAA AGA TCC GAT TGG AAC CGT CCC AAG CGT TGC G) was added TRITON linker with platinum group (Compound 2a, 1.2

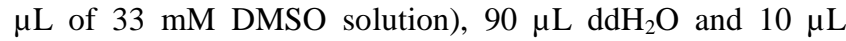
DMSO. The reaction mixture was stirred at $55^{\circ} \mathrm{C}$ for $30 \mathrm{~min}$. Then it was purified by Nap-5 column (GE Healthcare, USA) pre-equilibrated with $10 \mathrm{~mL} \mathrm{ddH}_{2} \mathrm{O}$. Degree of labeling was evaluated through UV VIS analysis with the dye main absorption band as chromophore (Rhodamine $6 \mathrm{G}, \lambda \max =541 \mathrm{~nm}$, molar extinction 116000), Biodrop instrument, the labeling ratio of Dye/oligonucleotide was evaluated as 1.74.

Labeling readout strands with DBCO-modified dyes. To a solution of $20 \mu \mathrm{L}$ azide-functionalized ssDNA $(0.1 \mathrm{mM}$ in $1 \mathrm{x}$ PBS, readout strand: /5AzideN/AAA AGA TCC GAT TGG AAC CGT CCC AAG CGT TGC G) was added $90 \mu \mathrm{L} 1 \times$ PBS, $10 \mu \mathrm{L}$ DMSO and DBCO-modified rhodamine B or rhodamine 6G (10 equiv.). The reaction mixture was stirred at $35^{\circ} \mathrm{C}$ for 1 hour, and resulting labeled oligonucleotides were purified by Nap-5 column (GE Healthcare, USA) using the manufacturer's

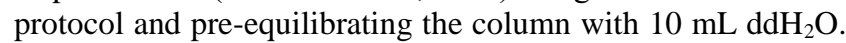
Degree of labeling was evaluated using the abovementioned methods.

Labeling encoding sequences of RNA. Degree of labeling was evaluated using the abovementioned methods.

Fluorescence imaging. Image acquisition was performed on an inverted Leica true confocal scanner SP8 X system (Wetzlar, Germany). Laser power (LP) is measured by an optical power meter (THORLABS) using a photodiode sensor (S170C, THORLABS). All pre-expansion images were acquired using a HCPLAPO CS2 63× water immersion objective (NA 1.2) and post-expansion images were achieved with Fluotar VISIR $25 \times$ water immersion objective (NA 0.95). Z-stacks of the RNA FISH images were processed using Image J/FIJI. Nuclear DAPI staining was imaged with a $405 \mathrm{~nm}$ pulsed diode laser. Other dyes were activated using A supercontinuum white light laser (SuperK EXTREME/FIANIUM, NKT photonics, Birkerød, Denmark). The laser power of the supercontinuum white light laser, the gain, and the pinhole size (1 airy unit (AU)) were kept constant when acquiring pre- and post-images.

Quantification of resolution improvement and RNA molecules. We imaged the same cellular areas pre and post expansion to verify the resolution improvement. To evaluate the expansion factor in $\times 4$ and $\times 10$ ExM, we measured the area of the same DAPI-stained nucleus before and after expansion using Fiji to calculate the expansion factor, and the experimental image resolution was determined using a line profile across stained microtubule filaments. A Gaussian curve was fitted to the line profile using the ImageJ FWHM_line plugin and these results were used to confirm image resolution. FWHM values obtained for post-ExM images were further corrected with the obtained expansion factor. For comparison, the theoretical resolution was calculated based on the Rayleigh criterion $\left(r=0,61 \times \lambda_{\text {emission }}\right.$ / NA). Counting RNA molecules of cells was performed using TrackMate Fiji plugin of Image J.

\section{ASSOCIATED CONTENT}

(Word Style "TE_Supporting_Information"). Supporting Information. The Supporting information is available free of charge on the ACS Publication website at DOI: . . Detailed experimental procedures, compound characterization data, tables and figures (PDF);

Video S1: Post-expansion z-stack of $P G K 1$ transcripts in singlecolor staining (AVI);

Video S2: Post-expansion z-stack of GAPDH transcripts in multicolor staining (AVI);

Video S3: Post-expansion z-stack of $P G K 1$ transcripts in multicolor staining (AVI).

\section{AUTHOR INFORMATION}

\section{Corresponding Author}

Johan Hofkens - Department of Chemistry, KU Leuven, Leuven, 3001, Belgium/ LISCO, KU Leuven Institute for Single-Cell Omics, Leuven, 3000, Belgium; orcid.org/0000-0002-9101-0567; Email: johan.hofkens@kuleuven.be.

Notes

The authors declare the following competing financial interest(s): J.H. holds shares of Chrometra, a company that commercializes TRITON linkers and some of the reagents described.

\section{ACKNOWLEDGMENTS}

This work was supported by funding from the China Scholarship Council to G.W. (Nr 201806210078), from the Flemish government through long-term structural funding Methusalem (CASAS2, Meth/15/04) to J.H., from FWO through fellowships to M.V. $(1 \mathrm{~S} 62318 \mathrm{~N})$, and from KU Leuven research fund through ID-N project IDN/19/039. The authors thank C. Jackers, R. Nuyts and A. De Weerdt for technical support.

\section{REFERENCES}

(1) Raj, A.; Van Oudenaarden, A. Nature, nurture, or chance: stochastic gene expression and its consequences. Cell 2008, 135 (2), 216226.

(2) Buxbaum, A. R.; Haimovich, G.; Singer, R. H. In the right place at the right time: visualizing and understanding mRNA localization. Nat. Rev. Mol. Cell Biol. 2015, 16 (2), 95-109.

(3) Crosetto, N.; Bienko, M.; Van Oudenaarden, A. Spatially resolved transcriptomics and beyond. Nat. Rev. Genet. 2015, 16 (1), 57 66.

(4) Femino, A. M.; Fay, F. S.; Fogarty, K.; Singer, R. H. Visualization of single RNA transcripts in situ. Science 1998, 280 (5363), 585590.

(5) Raj, A.; Van Den Bogaard, P.; Rifkin, S. A.; Van Oudenaarden, A.; Tyagi, S. Imaging individual mRNA molecules using multiple singly labeled probes. Nat. methods 2008, 5 (10), 877-879.

(6) Chen, K. H.; Boettiger, A. N.; Moffitt, J. R.; Wang, S.; Zhuang, $\mathrm{X}$. Spatially resolved, highly multiplexed RNA profiling in single cells. Science 2015, 348 (6233).

(7) Wang, G.; Moffitt, J. R.; Zhuang, X. Multiplexed imaging of high-density libraries of RNAs with MERFISH and expansion microscopy. Sci. Rep. 2018, 8 (1), 4847. 
(8) Chen, F.; Tillberg, P. W.; Boyden, E. S. Expansion microscopy. Science 2015, 347 (6221), 543-548.

(9) Chozinski, T. J.; Halpern, A. R.; Okawa, H.; Kim, H. J.; Tremel, G. J.; Wong, R. O.; Vaughan, J. C. Expansion microscopy with conventional antibodies and fluorescent proteins. Nat. methods 2016, 13 (6), 485-8.

(10) Tillberg, P. W.; Chen, F.; Piatkevich, K. D.; Zhao, Y.; Yu, C.C. J.; English, B. P.; Gao, L.; Martorell, A.; Suk, H.-J.; Yoshida, F. Protein-retention expansion microscopy of cells and tissues labeled using standard fluorescent proteins and antibodies. Nat. Biotechnol. 2016, 34 (9), 987-992.

(11) Chang, J.-B.; Chen, F.; Yoon, Y.-G.; Jung, E. E.; Babcock, H.; Kang, J. S.; Asano, S.; Suk, H.-J.; Pak, N.; Tillberg, P. W. Iterative expansion microscopy. Nat. methods 2017, 14 (6), 593-599.

(12) Halpern, A. R.; Alas, G. C. M.; Chozinski, T. J.; Paredez, A. R.; Vaughan, J. C. Hybrid Structured Illumination Expansion Microscopy Reveals Microbial Cytoskeleton Organization. ACS nano 2017, 11 (12), 12677-12686.

(13) Park, C. E.; Cho, Y.; Cho, I.; Jung, H.; Kim, B.; Shin, J. H.; Choi, S.; Kwon, S. K.; Hahn, Y. K.; Chang, J. B. Super-Resolution Three-Dimensional Imaging of Actin Filaments in Cultured Cells and the Brain via Expansion Microscopy. ACS nano 2020, 14 (11), 1499915010.

(14) Vanheusden, M.; Vitale, R.; Camacho, R.; Janssen, K. P.; Acke, A.; Rocha, S.; Hofkens, J. Fluorescence photobleaching as an intrinsic tool to quantify the 3D expansion factor of biological samples in expansion microscopy. ACS omega 2020, 5 (12), 6792-6799.

(15) Chen, F.; Wassie, A. T.; Cote, A. J.; Sinha, A.; Alon, S.; Asano, S.; Daugharthy, E. R.; Chang, J. B.; Marblestone, A.; Church, G. M.; Raj, A.; Boyden, E. S. Nanoscale imaging of RNA with expansion microscopy. Nat. methods 2016, 13 (8), 679-84.

(16) Saka, S. K.; Wang, Y.; Kishi, J. Y.; Zhu, A.; Zeng, Y.; Xie, W.; Kirli, K.; Yapp, C.; Cicconet, M.; Beliveau, B. J.; Lapan, S. W.; Yin, S.; Lin, M.; Boyden, E. S.; Kaeser, P. S.; Pihan, G.; Church, G. M.; Yin, P. Immuno-SABER enables highly multiplexed and amplified protein imaging in tissues. Nat. Biotechnol. 2019, 37 (9), 1080-1090.

(17) Tsanov, N.; Samacoits, A.; Chouaib, R.; Traboulsi, A. M.; Gostan, T.; Weber, C.; Zimmer, C.; Zibara, K.; Walter, T.; Peter, M.; Bertrand, E.; Mueller, F. smiFISH and FISH-quant - a flexible single RNA detection approach with super-resolution capability. Nucleic Acids Res. 2016, 44 (22), e165.

(18) Lubeck, E.; Coskun, A. F.; Zhiyentayev, T.; Ahmad, M.; Cai, L. Single-cell in situ RNA profiling by sequential hybridization. Nat. methods 2014, 11 (4), 360.

(19) Wen, G.; Vanheusden, M.; Acke, A.; Valli, D.; Neely, R. K.; Leen, V.; Hofkens, J. Evaluation of direct grafting strategies via trivalent anchoring for enabling lipid membrane and cytoskeleton staining in expansion microscopy. ACS nano 2020, 14 (7), 7860-7867.

(20) Fleet, G.; Porter, R.; Knowles, J. Affinity labelling of antibodies with aryl nitrene as reactive group. Nature 1969, 224 (5218), 511-512.

(21) Smith, R. A.; Knowles, J. R. Aryldiazirines. Potential reagents for photolabeling of biological receptor sites. J. Am. Chem. Soc. 1973, 95 (15), 5072-5073.

(22) Polavarapu, A.; Stillabower, J. A.; Stubblefield, S. G.; Taylor, W. M.; Baik, M.-H. The mechanism of guanine alkylation by nitrogen mustards: a computational study. J. Org. Chem. 2012, 77 (14), 59145921.

(23) Heetebrij, R.; Talman, E.; Velzen, M. v.; Gijlswijk, R. v.; Snoeijers, S.; Schalk, M.; Wiegant, J.; Rijke, F. v.; Kerkhoven, R.; Raap, A. Platinum (II)-Based Coordination Compounds as Nucleic
Acid Labeling Reagents: Synthesis, Reactivity, and Applications in Hybridization Assays. Chembiochem 2003, 4 (7), 573-583.

(24) Galardy, R. E.; Craig, L. C.; Jamieson, J. D.; Printz, M. P. Photoaffinity labeling of peptide hormone binding sites. J. Biol. Chem. 1974, 249 (11), 3510-3518.

(25) Wiegant, J.; Van Gijlswijk, R.; Heetebrij, R.; Bezrookove, V.; Raap, A.; Tanke, H. ULS: a versatile method of labeling nucleic acids for FISH based on a monofunctional reaction of cisplatin derivatives with guanine moieties. Cytogenet. Genome Res. 1999, 87 (1-2), 47-52.

(26) Sheldon, E. L.; Kellogg, D. E.; Watson, R.; Levenson, C. H.; Erlich, H. A. Use of nonisotopic M13 probes for genetic analysis: application to HLA class II loci. Proc. Natl. Acad. Sci. U. S. A.1986, 83 (23), 9085-9089.

(27) Albarella, J.; Minegar, R.; Patterson, W.; Dattagupta, N.; Carlson, E. Monoadduct forming photochemical reagents for labeling nucleic acids for hybridization. Nucleic Acids Res. 1989, 17 (11), 4293 4308.

(28) Bordin, F.; Carlassare, F.; Baccichetti, F.; Guiotto, A.; Rodighiero, P.; Vedaldi, D.; Dall'Acqua, F. 4, 5'-Dimethylangelicin: A New DNA-Photobinding Monfunctional Agent. Photochem. Photobiol. 1979, 29 (6), 1063-1070.

(29) Knowles, J. R. Photogenerated reagents for biological receptorsite labeling. Acc. Chem. Res. 1972, 5 (4), 155-160.

(30) Liang, T. Y.; Schuster, G. B. Photochemistry of 3-and 4-nitrophenyl azide: detection and characterization of reactive intermediates. J. Am. Chem. Soc. 1987, 109 (25), 7803-7810.

(31) Gotz, R.; Kunz, T. C.; Fink, J.; Solger, F.; Schlegel, J.; Seibel, J.; Kozjak-Pavlovic, V.; Rudel, T.; Sauer, M. Nanoscale imaging of bacterial infections by sphingolipid expansion microscopy. Nat. Commun. 2020, 11 (1), 6173

(32) van Belkum, A.; Linkels, E.; Jelsma, T.; Houthoff, H. J.; van Den Berg, F.; Quinta, W. Application of a new, universal DNA labeling system in the PCR mediated diagnoses of Chlamydia trachomatis and human papillomavirus type 16 infection in cervical smears. J. Virol. methods 1993, 45 (2), 189-200.

(33) Choi, H. M.; Schwarzkopf, M.; Fornace, M. E.; Acharya, A.; Artavanis, G.; Stegmaier, J.; Cunha, A.; Pierce, N. A. Third-generation in situ hybridization chain reaction: multiplexed, quantitative, sensitive, versatile, robust. Development 2018, 145 (12).

(34) Choi, H. M.; Beck, V. A.; Pierce, N. A. Next-generation in situ hybridization chain reaction: higher gain, lower cost, greater durability. ACS nano 2014, 8 (5), 4284-4294.

(35) Lin, R.; Feng, Q.; Li, P.; Zhou, P.; Wang, R.; Liu, Z.; Wang, Z.; Qi, X.; Tang, N.; Shao, F.; Luo, M. A hybridization-chain-reactionbased method for amplifying immunosignals. Nat. methods 2018, 15 (4), 275-278.

(36) Moffitt, J. R.; Hao, J.; Bambah-Mukku, D.; Lu, T.; Dulac, C.; Zhuang, X. High-performance multiplexed fluorescence in situ hybridization in culture and tissue with matrix imprinting and clearing. Proc. Natl. Acad. Sci. U. S. A. 2016, 113 (50), 14456-14461.

(37) Shah, S.; Lubeck, E.; Schwarzkopf, M.; He, T.-F.; Greenbaum, A.; Sohn, C. H.; Lignell, A.; Choi, H. M.; Gradinaru, V.; Pierce, N. A. Single-molecule RNA detection at depth by hybridization chain reaction and tissue hydrogel embedding and clearing. Development 2016, 143 (15), 2862-2867. 
1

2

3

4

5

6

7

8

9

10

11

12

13

14

15

16

17

18

19

20

21

22

23

24

25

26

27

28

29

30

31

32

33

34

35

36

37

38

39

40

41

42

43

44

45

46

47

48

49

50

51

52

53

54

55

56

57

58

59

60

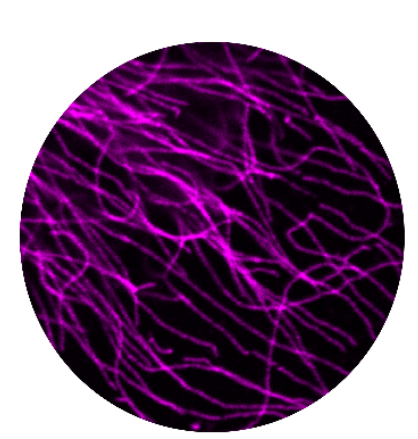

Versatile biolabeling for ExM
For TOC graphic

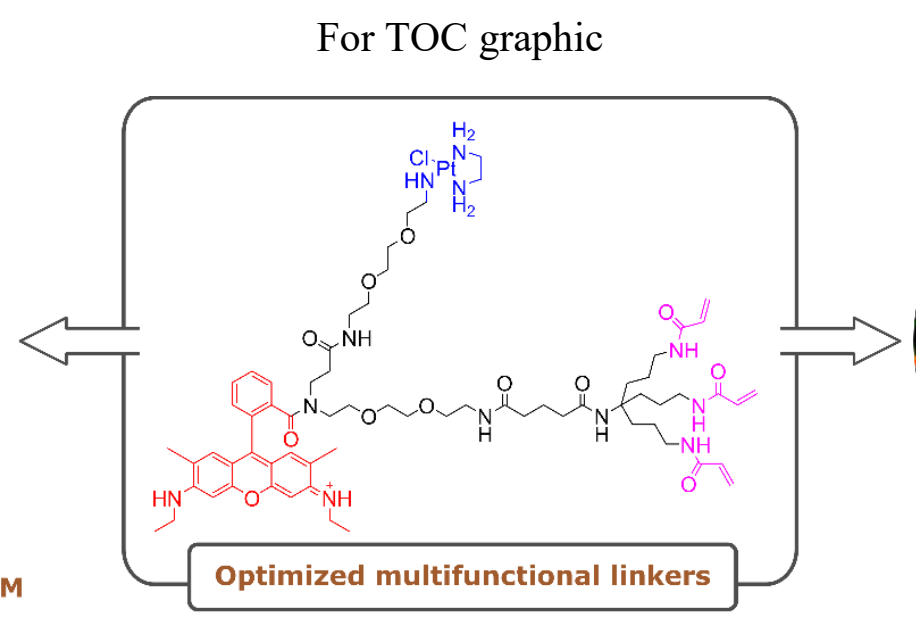

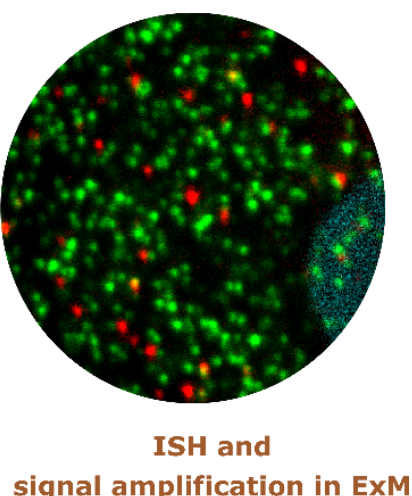

signal amplification in ExM 


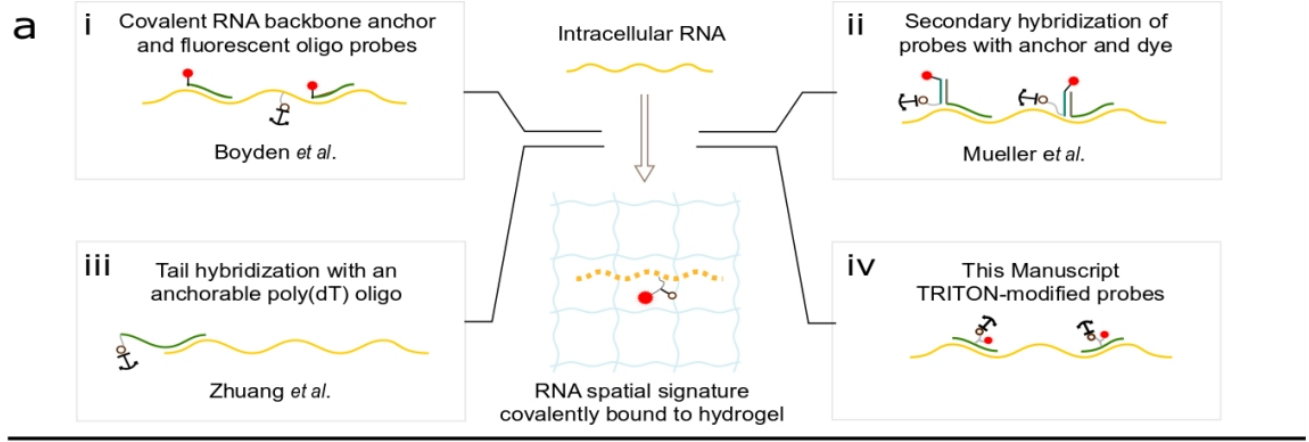

b

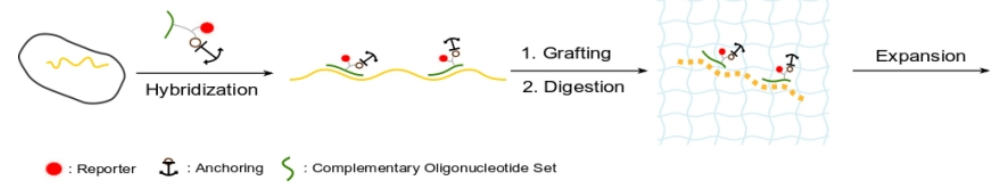

Figure 1. Schematic diagram of the different approaches for grafting and imaging ribonucleic acids within the ExM polymer. (a) The anchoring strategies of RNA via LabelX (i), acrydite-modified FISH probes (ii), acrydite-modified poly(dT) oligonucleotides for tail hybridization (iii) and our TRITON-modified encoding sequences (iv). (b) Workflow of our TRITON linkers used in ExM. Encoding sequences are labeled with our TRITON molecules in a single step approach.

$357 \times 193 \mathrm{~mm}(96 \times 96 \mathrm{DPI})$ 


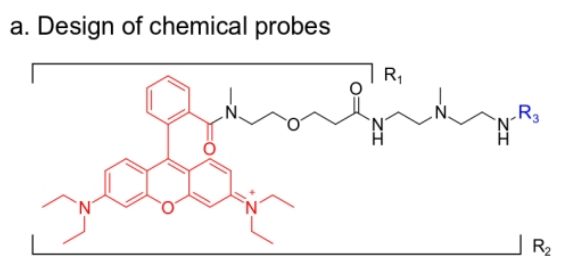

1) Photoaffinity probes
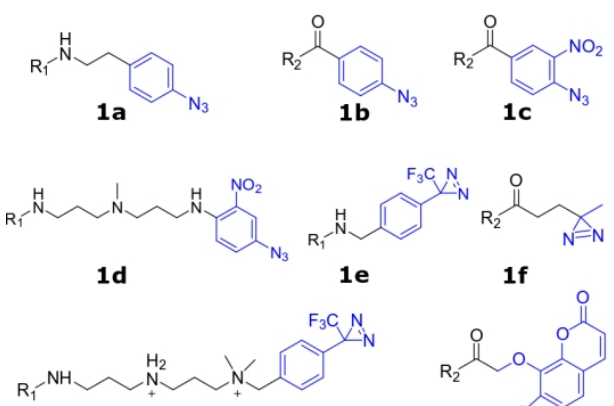

$1 \mathrm{~g}$
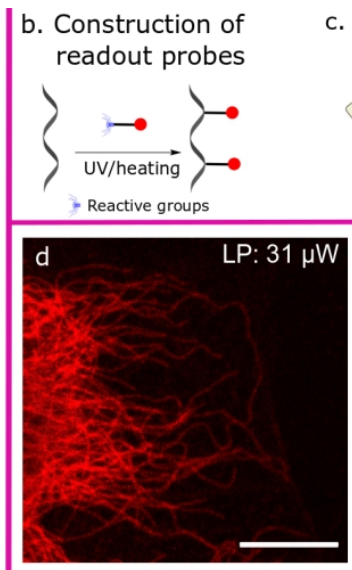

Labeling density: 1.21

Photoaffinity Probe 1d

c. Evaluation of hybridization efficiency
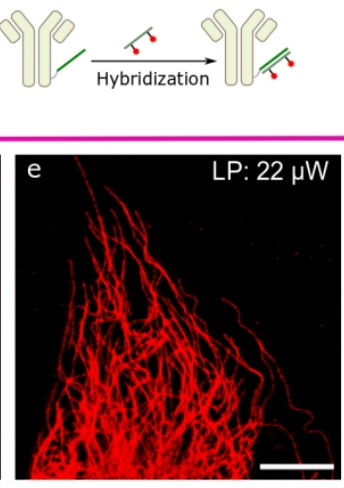

Labeling density: 3.00

1f

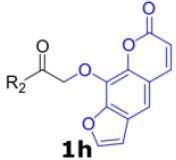

2) Thermalaffinity probes<smiles>[R]NCCOCCOCCN[NH+]1NCCN1Cl</smiles>

1i

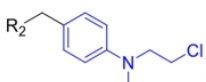

1j

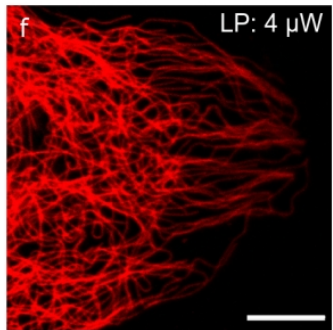

Labeling density: 1.58

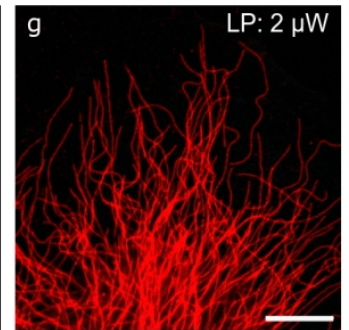

Labeling density: 2.57 Thermalaffinity Probe 1i

Figure 2. Design and optimization of chemical probes for nucleic acids. (a) Synthesis of a series of probes with different functional groups. (b) Schematic representation of the construction of readout probes. (c) Schematic diagram of evaluating hybridiza-tion properties via combination with oligonucleotide-labeled antibodies. ( $\mathrm{d}-\mathrm{g}$ ) Images obtained in immunostaining experi-ments with compound $1 \mathrm{~d}$ and $1 \mathrm{i}$. All experiments are per-formed in the same condition except the laser power used for imaging. Scale bars: 10 $\mu \mathrm{m}(\mathrm{d}, \mathrm{e}, \mathrm{f}, \mathrm{g})$.

$480 \times 374 \mathrm{~mm}(96 \times 96 \mathrm{DPI})$ 
b.
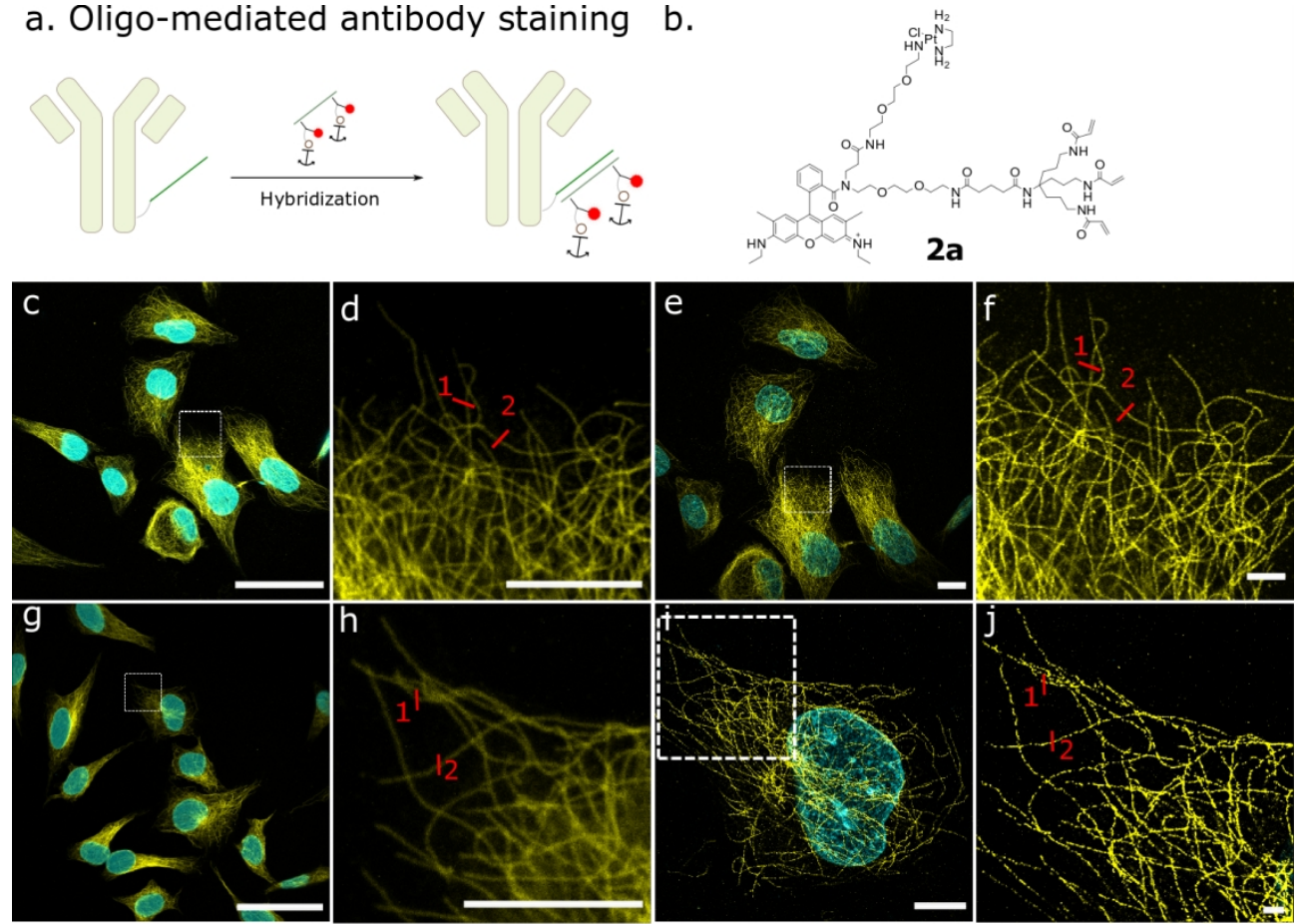

k. Chemical structures
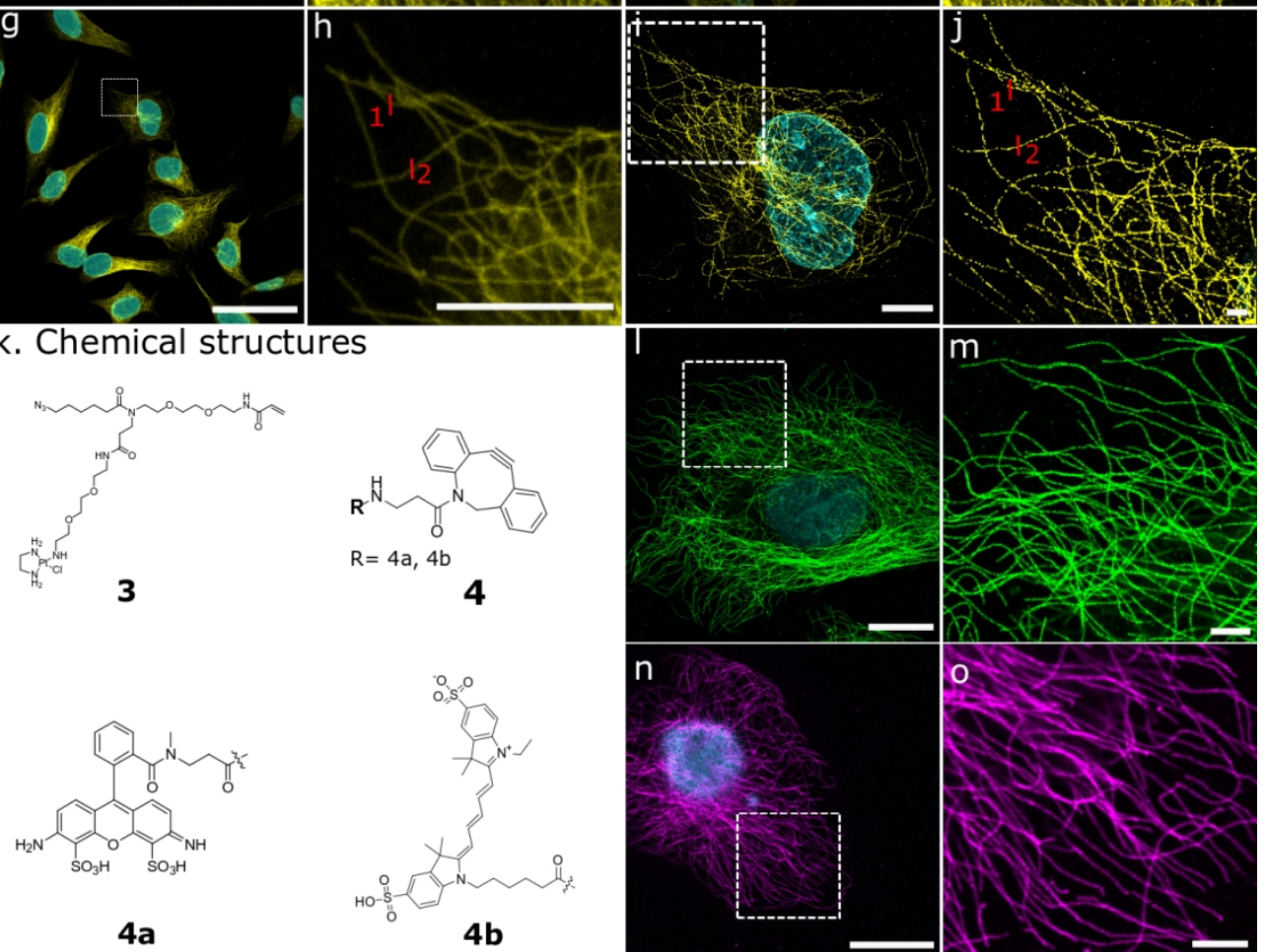

$4 a$

$4 b$
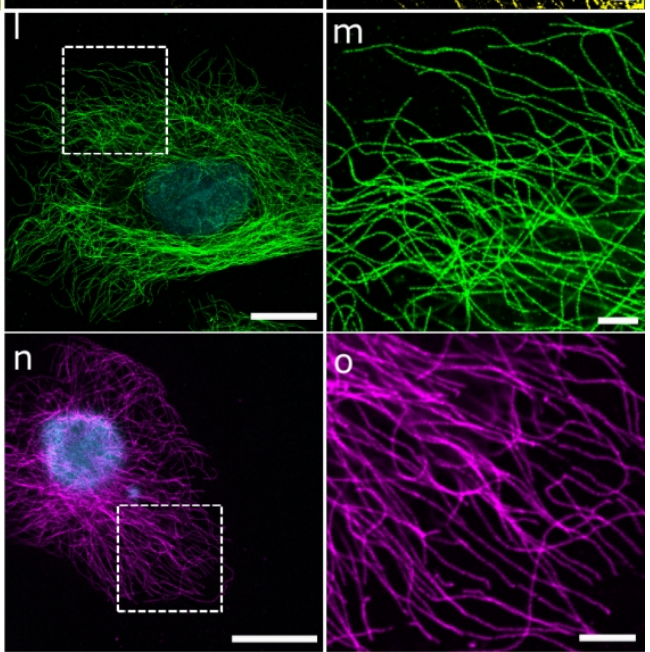

Figure 3: The immunostaining experiment using TRITON-based readout probes in $\times 4$ and $\times 10$ ExM. (a) Oligonucleotide-mediated immunostaining in which imager strands were labeled by our TRITON linker. (b) Chemical structure of TRITON link-er 2a. (c-f) Immunostaining against microtubules with TRITON linker $2 a$ (Rh 6G, platinum and acrylates) in $\times 4$ ExM. (c) Pre-expansion image obtained in $\times 4$ ExM. (d) Zoom of the high-lighted region in (c) and representative FWHM line profiles (1) $355 \mathrm{~nm}$ and (2) $229 \mathrm{~nm}$. (e) Postexpansion image obtained in $\times 4$ ExM. (f) Zoom of the highlighted region in (e) and repre-sentative FWHM line profiles (1) $419 \mathrm{~nm}$ and (2) 345nm. ( $\mathrm{g}-\mathrm{j}$ ) Immunostaining against microtubules with TRITON linker $2 \mathrm{a}$ in $\times 10$ ExM. (g) Pre-expansion image obtained in $\times 10$ ExM. (h) Zoom in on highlighted region in $(\mathrm{g})$ and representative FWHM line profiles (1) $269 \mathrm{~nm}$ and (2) $292 \mathrm{~nm}$. (i) Post-expansion image obtained in $\times 10$ ExM. (j) Zoom in on highlighted region in (i) and representative FWHM line profiles (1) $347 \mathrm{~nm}$ and (2) 407 $\mathrm{nm}$. (k) Chemical structures of compound 3 and 4 used in a post-digestion labeling strategy. (I) Postdigestion labeling image obtained with compound 3 and compound $4 a$ (Atto 488-DBCO) in $\times 4$ ExM. (m)

Zoom in on highlighted region in (I). (n) Post-digestion labeling image obtained with compound 3 and compound 4b (Sulfo-Cy5-DBCO) in $\times 4$ ExM. (o) Zoom in on highlighted region in ( $n$ ). Scale bars: $50 \mu m(c$, 
$e, g, i, l, n), 10 \mu m(d, f, h, j, m, o)$.

$403 \times 490 \mathrm{~mm}(96 \times 96$ DPI) 
a
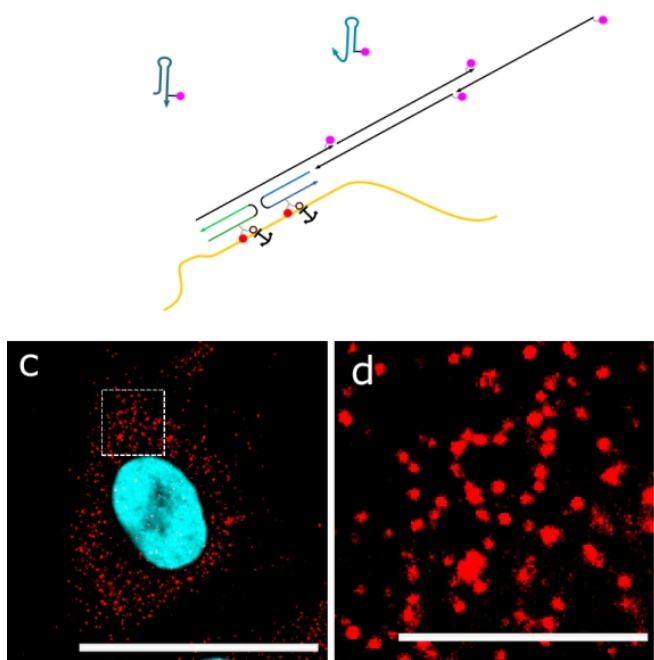

g
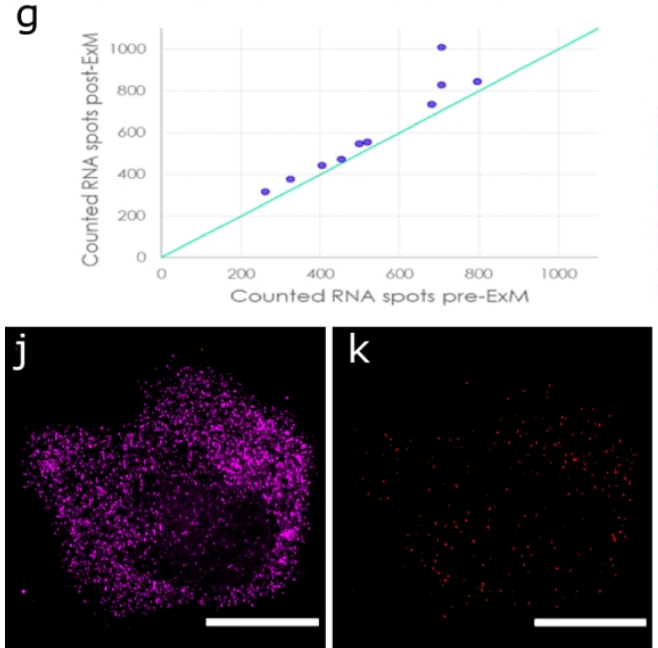

b

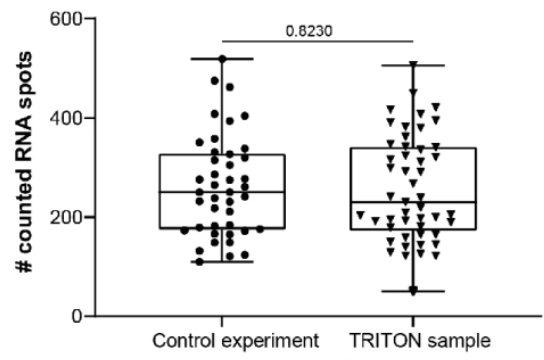

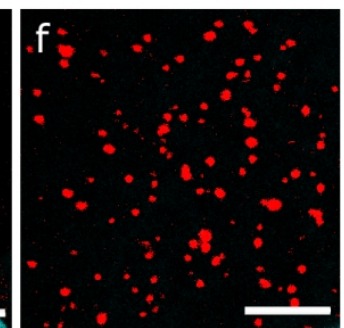
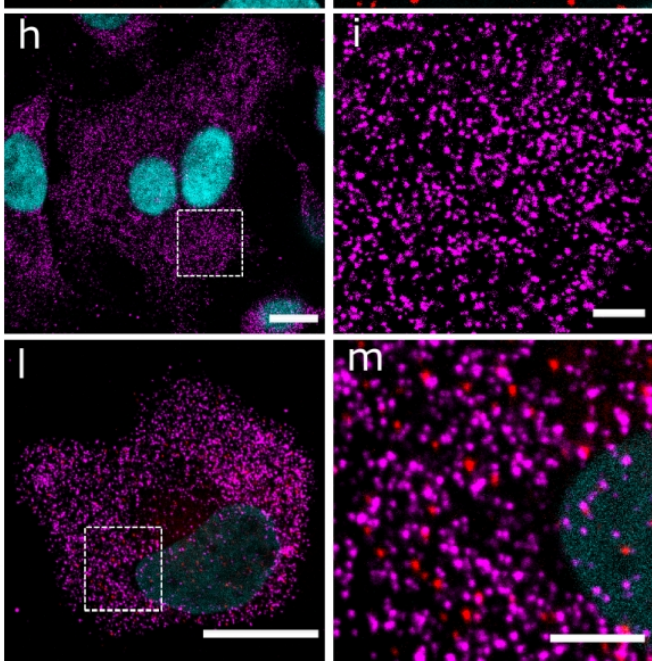

Figure 4. mRNA detection via combining ExM with HCR v3.0 using TRITON linker 2a. (a) Design of HCR v3.0mediated signal amplification. TRITON-modified FISH probes first hy-bridize to the target mRNA and metastable DNA hairpins bear-ing fluorophores then bind to the split initiators, thus triggering signal amplification. (b) Estimated number of PGK1 transcripts per cell in a control experiment and our TRITON samples in three independent experiments. The $\mathrm{P}$ value is listed, and $\mathrm{P}<0.05$ is considered significant. Control experiment: Commercial encoding probes (no TRITON linker $2 a$ modification) directly bind to PGK1mRNA and Alexa Fluor 594-labeled hairpins then bind to the split initiators to trigger signal amplification. (c-

f) Images of PGK1 before and after expansion, using Alexa Fluor 594-labeled hairpins. (c) Pre-expansion image of PGK1 transcripts. (d) Zoom in on highlighted region in (c). (e) Post-expansion image of the same cell. (f) Zoom in on highlighted region in (e). (g) mRNA molecules counted before versus after expansion for cells from 3 independent samples $(n=10)$. The cyan line is used as a guide to prove the loss of PGK1 transcripts detected in unexpanded samples. (h) Post-labeling image of GAPDH transcripts, using Alexa Fluor 647-labeled hairpins. (i) Zoom in on highlighted region in (h). (j-m) Multi-color stain-ing against PGK1 and GAPDH transcripts in Hela cells (PGK1: Alexa Fluor 594-labeled hairpins; GAPDH: Alexa Fluor 647-labeled hairpins); nuclei were marked with DAPI. (j) Image of GAPDH. (k) Image of PGK1. (I) Merge image of GAPDH and PGK1. (m) Zoom in on highlighted region in (I). Scale bars: $50 \mu \mathrm{m}(c, e, h, j, k, l), 10 \mu \mathrm{m}(d, f$, $\mathrm{i}, \mathrm{m})$. 
